Relations industrielles

Industrial Relations

\title{
Personnel: A Diagnostic Approach, 3d edition, par William F. Glueck, Business Publications Inc., 1982, 720 pp., ISBN 0-256-02429-4.
}

\section{Laurent Bélanger}

Volume 37, numéro 2, 1982

URI : https://id.erudit.org/iderudit/029271ar

DOI : https://doi.org/10.7202/029271ar

Aller au sommaire du numéro

Éditeur(s)

Département des relations industrielles de l'Université Laval

ISSN

0034-379X (imprimé)

1703-8138 (numérique)

Découvrir la revue

Citer ce compte rendu

Bélanger, L. (1982). Compte rendu de [Personnel: A Diagnostic Approach, 3d edition, par William F. Glueck, Business Publications Inc., 1982, 720 pp., ISBN 0-256-02429-4.] Relations industrielles / Industrial Relations, 37(2), 459-460. https://doi.org/10.7202/029271ar

Tous droits réservés @ Département des relations industrielles de l'Université Laval, 1982
Ce document est protégé par la loi sur le droit d'auteur. L'utilisation des services d'Érudit (y compris la reproduction) est assujettie à sa politique d'utilisation que vous pouvez consulter en ligne.

https://apropos.erudit.org/fr/usagers/politique-dutilisation/ 
cette méthode est une thérapie individuelle fondée sur l'intérêt personnel et la stabilité émotive du gestionnaire et elle repose sur l'acceptation de soi-même, compte tenu de ses limites et des réalités de son environnement. Cette méthode, conçue à l'origine pour corriger des perturbations de comportement, trouve, d'après l'auteur, des applications intéressantes dans la gestion des entreprises.

Le gestionnaire est invité à regarder la réalité en face, à reconnaître ses forces, ses faiblesses et à bien identifier les événements et leur environnement. Les références fréquentes aux travaux de Peter Drucker et à ceux du docteur Lucien Auger du Centre interdisciplinaire de Montréal traduisent la source des influences qui ont marqué l'auteur.

Celui-ci fait cheminer le gestionnaire, chapitre après chapitre, selon un même cadre pédagogique. Il lui fait d'abord découvrir les objectifs de l'entreprise et l'invite à situer son action à l'intérieur de ce cadre; il l'incite au réalisme personnel et au développement de ses propres valeurs dans le respect des valeurs et des capacités de ses collègues; dans cette veine, il valorise une communication avec ces derniers fondée sur le respect mutuel; l'évaluation des subordonnés et les sanctions consécutives de même que la relation avec les patrons s'inscrivent dans la même pensée.

À une époque où plusieurs s'interrogent sur la façon de gérer, où bien des modes et des façons de faire sont remises en cause, cet ouvrage constitue une pièce à examiner attentivement et représente une contribution intéressante à ce débat.

On regrette cependant de ne pas y trouver de données mesurables sur l'efficacité de la méthode en question. Il est à souhaiter que l'auteur procède à l'élaboration de mesures quantifiables permettant d'évaluer cette thérapie. De même, on peut s'interroger sur l'absence de références à des comportements collectifs ou de groupe dans l'entreprise résultant notamment de l'action syndicale ou des associations. Cela est toutefois bien compréhensible car la méthode "émotivo-rationnel- le» est fondée essentiellement sur l'intérêt personnel et le désir de chacun de réussir.

\section{Bernard ANGERS}

Gouvernement du Québec

Personnel: A Diagnostic Approach, 3d edition, par William F. Glueck, Business Publications Inc., 1982, 720 pp., ISBN 0-256-02429-4.

Bill Glueck est un de mes confrères de classe décédé récemment avant même d'avoir terminé la rédaction de la troisième édition de cet ouvrage publié en 1974 et portant le même titre. C'était un type qui savait allier une grande capacité de synthèse à la minutie du détail. George T. Milkovich s'est chargé de superviser la révision et la rédaction de cette troisième édition et il en a également écrit la préface.

L'ouvrage retient le modèle de base tiré de l'approche systémique que Bill Glueck avait été le premier à présenter d'une façon articulée et opérationnelle en matière de gestion opérationnelle et intégrée des ressources humaines au sein des organisations du travail. L'approche systémique et diagnostique permet d'abord de bien saisir la double finalité de la fonction ressources humaines et sa relation étroite avec les objectifs généraux et opérationnels d'une organisation. Une telle approche permet ensuite de saisir le degré de cohérence interne au sein de l'ensemble des activités regroupées sous cette fonction, de même que l'impact des facteurs d'environnement internes à l'organisation et des facteurs d'environnement externes sur l'accomplissement de l'une ou l'autre des activités. Le modèle systémique adopté comprend donc les quatre grandes composantes suivantes: des facteurs d'environnement externes tels que le contexte économique, politique, législatif et culturel; des facteurs internes à l'organisation tels que les objectifs, la nature des tâches, les caractéristiques des individus et des groupes appelés à les accomplir; des activités spécifiques à la gestion des ressources humaines 
dont on retrouve la description détaillée dans d'autres bons manuels; enfin, les finalités de la fonction et les indicateurs de mesure de la performance. Chaque partie de l'ouvrage et les chapitres qu'il contient se greffent sur ces quatre grandes composantes: ce qui en fait un volume d'une lecture facile surtout pour ceux qui veulent s'initier à ce champ de connaissances et de pratiques administratives. Chaque chapitre débute avec un rappel du modèle initial et situe le sujet qui sera traité au confluent des interdépendances entre l'activité en question et les variables externes ou internes qui l'influencent.

Les références à la fin de chaque chapitre portent sur tous les points abordés et elles sont abondantes et à jour, tellement que la qualité principale de cette réédition est juste-

L'évolution des systèmes de travail dans l'économie moderne, Paris, Éditions du CNRS, 1981, 287 pp., ISBN 2-222-02935-X.

Ce volume comprend les Actes d'un Colloque organisé par le CEREQ à l'occasion de ses dix ans d'existence. Cinq thèmes y ont été développés. Économie et systèmes de travail: le travail et les performances économiques des entreprises et des nations; Technologie et systèmes de travail: évolution du travail face au développement des technologies; Systèmes de travail et localisation des emplois: les modifications dans les répartitions sectorielles et territoriales des emplois; Population et systè- ment ce répertoire bibliographique qui peut, si on possède bien la langue anglaise, épargner un nombre considérable d'heures de recherches à ceux qui veulent étudier plus en profondeur chacun des points abordés. Je m'en voudrais aussi de ne pas signaler la multitude des comparaisons faites entre le Canada et les États-Unis tout au long de cet ouvrage et la bibliographie en tient également compte. C'est donc à regret que je dois constater le décès vraiment prématuré de Bill, mais je sais qu'il laisse entre les mains de tous les étudiants et praticiens un instrument fort valable.

\section{Laurent BÉLANGER}

Université Laval

mes de travail: les transformations de la population active et leurs rapports avec l'évolution du travail; La modernisation des formations: des nouveaux objectifs pour la formation professionnelle.

Comme les organisateurs du Colloque ont fait appel aux meilleurs spécialistes de France tant pour présenter les thèmes que pour les discuter, ce document dresse un tableau fort intéressant en même temps que révélateur de l'évolution récente des recherches socio-économiques en matière d'emploi.

Gérard DION

Université Laval 